\title{
Right ventricular outflow tract reintervention after primary tetralogy of Fallot repair in neonates and young infants
}

\author{
Shyamasundar Balasubramanya, MD, ${ }^{\mathrm{a}}$ David Zurakowski, MS, $\mathrm{PhD},{ }^{\mathrm{b}}$ Michele Borisuk, NP, ${ }^{\mathrm{a}}$ \\ Aditya K. Kaza, MD, ${ }^{a}$ Sitaram M. Emani, MD, ${ }^{a}$ Pedro J. del Nido, MD, ${ }^{a}$ and Christopher W. Baird, MD $^{\text {a }}$
}

\section{ABSTRACT}

Objective: To assess the outcomes following primary tetralogy of Fallot (TOF) repair in neonates and young infants with pulmonary stenosis (PS) and pulmonary atresia and compare differences in reintervention on the right ventricular outflow tract (RVOT) among those undergoing valve sparing repair (VSR), transannular RVOT patch (TAP), and right ventricle-to-pulmonary artery (RV-PA) conduit surgeries.

Methods: Data were collected retrospectively in 101 patients who underwent TOF repair over a 10-year period between January 2005 and September 2015. The primary endpoint was reintervention on the RVOT, defined as a surgical procedure or cardiac catheterization-based RVOT reintervention.

Results: Forty-three patients had TOF/PS, of whom $24(56 \%)$ underwent TAP and $19(44 \%)$ underwent VSR. Fifty-eight patients had TOF/PA, $14(24 \%)$ underwent TAP and $44(76 \%)$ underwent RV-PA conduit repair. Overall patient mortality was $2.9 \%$ ( 3 of 101). Thirty-three patients underwent surgical reintervention, and 52 underwent catheterization-based reintervention. Patients with TOF/PA who underwent RV-PA conduit repair had a higher surgical reintervention rate than those who underwent TAP (45\% vs $21 \%$ ). Patients with TOF/PSs undergoing VSR with a lower median birth weight $(2.5 \mathrm{~kg}$ vs $3.7 \mathrm{~kg})$ required more surgical reintervention.

Conclusions: Neonatal TOF repair can be performed with low mortality but frequent RVOT reinterventions. Surgical reintervention is earlier and the rate is higher among patients with TOF/PA undergoing RV-PA conduit repair compared with those undergoing TAP. Although there were no overall differences in RVOT reintervention rate between patients with TOF/PS undergoing VSR and those undergoing TAP, a lower birth weight in the patients undergoing VSR is associated with a higher surgical reintervention rate. ( $J$ Thorac Cardiovasc Surg 2018;155:726-34)

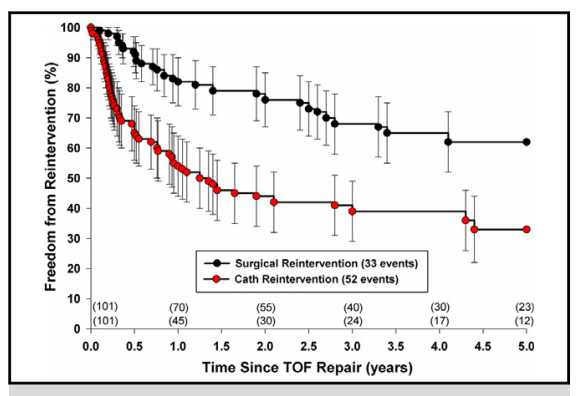

Kaplan-Meier curves showing freedom from first time reintervention in TOF(PS/PA) patients.

\section{Central Message}

Reintervention rates following TOF repair in neonates and young infants vary by weight and RVOT repair type, impacting the surgical RVOT approach and follow-up.

\section{Perspective}

Management of hypoxic neonates and young infants with TOF is challenging, with controversy regarding initial surgical strategy. Neonatal TOF repair can be performed with low mortality, but a frequent need for RVOT reintervention. Among patients with TOF/PA, the rate of reintervention is higher after RV-PA conduit repair than after TAP. In patients with TOF/PS, the rate of RVOT reintervention is comparable in patients undergoing VSR and those undergoing TAP; however, in VSR, low birth weight is a risk factor for reintervention.

See Editorial Commentaries pages 735 and 737.
From the Departments of ${ }^{\mathrm{a}}$ Cardiac Surgery and ${ }^{\mathrm{b}}$ Anesthesia, Boston Children's Hospital, Harvard Medical School, Boston, Mass.

Read at the 96th Annual Meeting of The American Association for Thoracic Surgery, Baltimore, Maryland, May 14-18, 2016.

Received for publication June 2, 2016; revisions received Aug 17, 2017; accepted for publication Sept 11, 2017; available ahead of print Oct 17, 2017.

Address for reprints: Shyamasundar Balasubramanya, MD, Department of Cardiac Surgery, Boston Children's Hospital, Harvard Medical School, 300 Longwood Ave, Bader 273, Boston, MA 02115 (E-mail: shyamabala@gmail.com). $0022-5223 / \$ 36.00$

Copyright @ 2017 Published by Elsevier Inc. on behalf of The American Association for Thoracic Surgery

https://doi.org/10.1016/j.jtcvs.2017.09.019
The first surgical treatment for Tetralogy of Fallot (TOF) was the palliative Blalock-Taussig (BT) shunt reported in 1945 by Blalock and Taussig, ${ }^{1}$ and the first successful complete primary intracardiac repair was reported by Lillehei and colleagues ${ }^{2}$ in 1954 using human cross-circulation. In the 1980s, Castaneda and colleagues ${ }^{3}$ and Barratt-Boyes

Scanning this QR code will take you to the article title page. 


$$
\begin{aligned}
& \text { Abbreviations and Acronyms } \\
& \text { BT }=\text { Blalock-Taussig } \\
& \text { CI }=\text { confidence interval } \\
& \text { ECMO }=\text { extracorporeal membrane oxygenation } \\
& \text { HR }=\text { hazard ratio } \\
& \text { ICU }=\text { intensive care unit } \\
& \text { IQR }=\text { interquartile range } \\
& \text { LOS }=\text { length of stay } \\
& \text { PS }=\text { pulmonary stenosis } \\
& \text { RVOT }=\text { right ventricular outflow tract } \\
& \text { RV-PA }=\text { right ventricle to pulmonary artery } \\
& \text { TAP }=\text { transannular patch } \\
& \text { TOF }=\text { tetralogy of Fallot } \\
& \text { VSR }=\text { valve-sparing repair }
\end{aligned}
$$

and Neutze ${ }^{4}$ demonstrated that primary repair could be achieved in infancy with excellent results. With improved outcomes over the past 6 decades, now $>95 \%$ of children born with TOF are expected to survive into adulthood, and over the last 2 decades, early primary repair has been extended to the cyanotic neonate. ${ }^{7-12}$ The goals of this single-center series were to assess outcomes following primary neonatal TOF repair in patients with pulmonary stenosis (PS) and pulmonary atresia, and to compare differences in reintervention on the right ventricular outflow tract (RVOT) among patients undergoing valve-sparing repair (VSR), transannular RVOT patch (TAP) surgery, or right ventricle-to-pulmonary artery (RV-PA) conduit repair.

\section{METHODS}

This was an Institutional Review Board-approved retrospective review of all 101 patients with congenital heart disease with PS or pulmonary atresia who underwent TOF repair who underwent TOF repair before 33 days of age between January 2005 and September 2015 at Boston Children's Hospital. The primary endpoint was reintervention on the RVOT, defined as a surgical procedure or a cardiac catheterization-based intervention on the RVOT.

Patients with TOF/PS underwent either pulmonary VSR or TAP, and patients with TOF/PA underwent either TAP or RV-PA conduit repair. All other primary surgical interventions were excluded from our analysis. Demographic data included date of birth, sex, and intraoperative height, body weight, and body surface area. Patient history data included the primary diagnosis and previous interventions, including catheterizations. Surgical data were obtained from the operative notes. Variables of interest include the date of admission, the date of initial operative repair, indication for operation, date of discharge, morbidity, and any complications before discharge. In addition to the specific RVOT approach, any concomitant procedures were recorded.

The closing date of follow-up for this study was April 1, 2016. Followup dates included the dates of most recent follow-up, echocardiography reports, and/or catheterization reports obtained as part of routine examinations. More specifically, data from echocardiography reports included date of echocardiography, estimated right ventricular pressure, maximum RVOT pressure gradient, and degrees of PS, pulmonary regurgitation, tricuspid regurgitation, right ventricular dysfunction, and left ventricular dysfunction.

\section{Statistical Methods}

Univariate analyses based on comparing binary proportions regarding reintervention were compared using Fisher's exact test, and categorical data were compared using Pearson's $\chi^{2}$ test. Patient characteristics; baseline continuous data, such as pulmonary valve $z$-score and RVOT gradient; and follow-up duration are expressed using median and interquartile range (IQR) because they were found to depart from a normal distribution by the Kolmogorov-Smirnov test, and groups were compared using the Mann-Whitney $U$-test. Freedom from reintervention was estimated using the Kaplan-Meier product-limit method, and surgical groups (VSR, TAP, and RV-PA conduit) were compared within each diagnosis (TOF/PS or TOF/PA) using the log-rank test. ${ }^{13}$ Greenwood's formula was used to construct $95 \%$ confidence intervals (CIs) around freedom from catheterization-based and surgical reintervention curves and overall patient survival. ${ }^{14}$ Median birth weight at the time of repair for patients with and without reintervention was compared using the Mann-Whitney $U$ test within each of the 3 surgical groups. The candidate variables that were considered in the multivariable analysis included birth weight, age $<14$ days at surgery, weight $<3 \mathrm{~kg}$, era of operation (before or after 2010), RVOT gradient, hospital length of stay (LOS) $>21$ days, and RVOT repair type. The number of candidate variables was limited to ensure that the regression coefficients remained stable without overfitting the model. ${ }^{15}$ Median intensive care unit (ICU) and hospital LOSs were compared among the VSR, TAP, and RV-PA conduit groups using the nonparametric Kruskal-Wallis test.

A Cox proportional hazards regression model was used to identify predictors of reintervention. The multivariable analysis included testing 2-way interactions to assess whether any possible risk factors were dependent on the type of surgical repair. ${ }^{16}$ The modulated renewal theory was applied using the nonparametric Nelson-Aalen estimator to derive the cumulative hazard function to compare the risk of catheterization-based reintervention incorporating all repeated episodes of catheterization-based reintervention for the same patient to assess differences between VSR $(n=19)$ and TAP $(n=24)$ in patients with TOF/PS and between RV-PA conduit repair $(n=44)$ and TAP $(n=14)$ in patients with TOF/PA. This assumes that the hazard for the time intervals between repeated or successive events for the same patient depends on the failure number via a covariate process and is suitable when the process can be expressed in terms of gaps between failure times instead of the total observation time. ${ }^{16}$ Thus, each segment has a beginning time and an ending time. Analyses were performed using R with the "survival" package ( $\mathrm{R}$ version 2.41-3; R Project for Statistical Computing, Vienna, Austria) using the survfit and coxph functions. All reported $P$ values are 2-tailed with a type I error of .05 as the criterion for statistical significance. All statistical analyses were conducted using SPSS version 23.0 (IBM, Armonk, NY).

\section{Definitions}

Early surgical reintervention on the RVOT was defined as any surgery performed on the RVOT after the initial surgery for TOF that addressed the RVOT or the RV-PA conduit and included RVOT muscle bundle resection, TAP, RV-PA conduit replacement, or pulmonary artery reintervention. Late surgical reintervention was defined as pulmonary valve replacement. Nonsurgical reintervention was defined as any intervention (1 or more) performed in the cardiac catheterization laboratory on the pulmonary arteries, RVOT, or RV-PA conduit, including balloon dilation or stent placement.

\section{RESULTS}

\section{Patient Characteristics}

Patient characteristics are summarized by TOF subtype in Table 1 and by initial surgical approach on the RVOT in Table 2. Of the 101 patients undergoing TOF repair between 2005 and 2015, 43 had TOF/PS and 58 had TOF/ PA. The median patient age at surgery was 11 days (IQR, 
TABLE 1. Baseline patient characteristics by diagnosis

\begin{tabular}{|c|c|c|c|c|}
\hline Variable & Overall $(n=101)$ & TOF/PS $(n=43)$ & TOF/PA $(n=58)$ & $P$ value \\
\hline \multicolumn{5}{|l|}{ Type of RVOT repair, n (\%) } \\
\hline VSR & $19(18.8)$ & 19 & - & \\
\hline TAP & $38(37.6)$ & 24 & 14 & \\
\hline RV-PA conduit & $44(43.5)$ & - & 44 & \\
\hline Age at primary surgery, $d$, median (IQR) & $11(4.0-20)$ & $17(11-28)$ & $6(3-12)$ & $<.001^{*}$ \\
\hline Age $<14 \mathrm{~d}$ at primary surgery, n (\%) & $61(61)$ & $27(63)$ & $45(78)$ & $<.001^{*}$ \\
\hline Male sex, n (\%) & $52(52)$ & $23(54)$ & $29(54)$ & .84 \\
\hline Weight at primary surgery, kg, median (IQR) & $2.9(2.5-3.5)$ & $3.0(2.5-3.7)$ & $2.9(2.4-3.3)$ & .14 \\
\hline PV annular size, mm, median (IQR) & - & $47(40-54)$ & - & \\
\hline PV $z$-score, median (IQR) & - & $-2.5(-3.3$ to -2.2$)$ & - & \\
\hline Preoperative RVOT gradient, mmHg, median (IQR) & - & $50(42-70)$ & - & \\
\hline \multicolumn{5}{|l|}{ Year of surgery, $\mathrm{n}(\%)$} \\
\hline Before 2010 & $48(48)$ & $19(44)$ & $29(50)$ & \\
\hline 2010 or later & $52(52)$ & $25(56)$ & $29(50)$ & \\
\hline
\end{tabular}

TOF/PS, Tetralogy of Fallot/pulmonary stenosis; TOF/PA, tetralogy of Fallot/pulmonary atresia; RVOT, right ventricular outflow tract; VSR, valve-sparing repair; TAP, transannular patch; $R V$ - $P A$, right ventricle to pulmonary artery; $I Q R$, interquartile range; $P V$, pulmonary valve. * Statistically significant difference $(P<.05)$.

4-20 days), and $61 \%$ of the patients were under 14 days of age. The median patient weight at surgery was $2.9 \mathrm{~kg}$ (IQR, $2.5-3.7 \mathrm{~kg}$ ). There was no significant difference in the sex distribution (52\% male). Nearly one-half of all patients underwent surgery in 2010 or later, but $85 \%$ of the TOF/PS VSR operations were performed in 2010 or later. The median preoperative RVOT gradient in the TOF/PS patients was $50 \mathrm{mmHg}$ (IQR, $42-70 \mathrm{mmHg}$ ) and was not different between the VSR and TAP groups $(P=.56)$. The median pulmonary valve $z$-score in the patients with TOF/PS was -2.5 , and was significantly lower in those who underwent TAP than in those who underwent VSR $(-3.1$ vs -2.3 ; $P<.001)$.

\section{RVOT Reintervention Rates for all Patients}

The median duration of follow-up was 3.5 years (IQR, 1.0-7.5 years), and differed by RVOT repair type, with a median follow-up of 2.2 years for VSR, 4.1 years for TAP, and 3.4 years for RV-PA conduit repair $(P=.06$, Kruskal-Wallis test). Among the entire cohort of 101, 59 patients underwent reintervention, including 52 with catheterization-based reintervention and 33 with surgical RVOT reintervention. The median time to first catheterization-based reintervention was 1.4 years $(95 \%$ CI, 6 months to 2.2 years), and that for first surgical reintervention was 7.1 years $(95 \% \mathrm{CI}, 4.9$ to 9.4 years) (Figure 1). One-half of the patients undergoing

TABLE 2. Baseline patient characteristics by type of RVOT repair

\begin{tabular}{|c|c|c|c|c|c|}
\hline Variable & Overall $(\mathbf{n}=101)$ & $\operatorname{VSR}(n=43)$ & $\begin{array}{c}\text { TAP } \\
(n=58)\end{array}$ & $\begin{array}{l}\text { RV-PA conduit } \\
\quad(n=44)\end{array}$ & $P$ value \\
\hline \multicolumn{6}{|l|}{ Diagnosis, n (\%) } \\
\hline TOF/PS & $43(42.5)$ & $19(44)$ & $24(56)$ & 0 & \\
\hline TOF/PA & $58(57.4)$ & 0 & $14(24)$ & $44(76)$ & \\
\hline Age at primary surgery, $d$, median (IQR) & $11(4.0-20)$ & $16(12-28)$ & $14(6-23)$ & $6(4-12)$ & $<.001 *$ \\
\hline Age $<14 \mathrm{~d}$ at primary surgery, $\mathrm{n}(\%)$ & $61(61)$ & $8(42)$ & $19(50)$ & $34(77)$ & $.008^{*}$ \\
\hline Male sex, $\mathrm{n}(\%)$ & $52(52)$ & $14(74)$ & $18(47)$ & $20(46)$ & .1 \\
\hline Weight at primary surgery, $\mathrm{kg}$, median (IQR) & $2.9(2.5-3.5)$ & $3.4(2.5-3.9)$ & $2.8(2.5-3.3)$ & $3.0(2.3-3.4)$ & .2 \\
\hline PV annular size, mm, median (IQR) & - & $52(45-64)$ & $44(35-48)$ & - & $.003^{*}$ \\
\hline PV z-score, median (IQR) & - & $-2.3(-2.9$ to -1.7$)$ & $-3.1(-3.9$ to -2.5$)$ & - & $.001 *$ \\
\hline Preoperative RVOT gradient, mm Hg, median (IQR) & $50(42-70)$ & $50(43-65)$ & $53(38-74)$ & - & .56 \\
\hline \multicolumn{6}{|l|}{ Year of surgery, $n(\%)$} \\
\hline Before 2010 & $48(48)$ & $3(15)$ & $24(63)$ & $21(48)$ & \\
\hline 2010 or later & $52(52)$ & $16(85)$ & $14(37)$ & $23(52)$ & \\
\hline
\end{tabular}

$V S R$, Valve-sparing repair; TAP, transannular patch; RV-PA, right ventricle to pulmonary artery; TOF/PS, tetralogy of Fallot/pulmonary stenosis; TOF/PA, tetralogy of Fallot/ pulmonary atresia; $I Q R$, interquartile range; $P V$, pulmonary valve; $R V O T$, right ventricular outflow tract. * Statistically significant differences $(P<.05)$. 


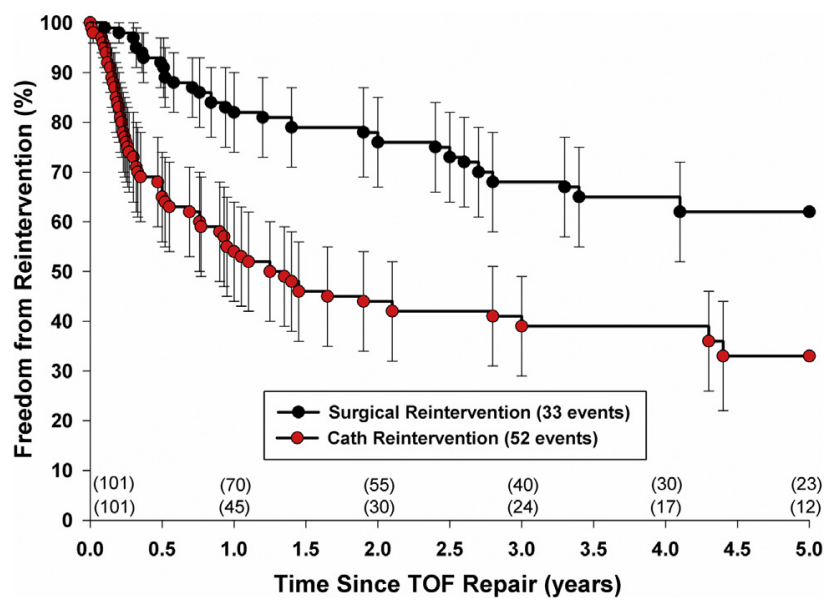

FIGURE 1. Kaplan-Meier curves showing the freedom from catheterization-based reintervention and surgical reintervention in the entire cohort of 101 patients. There were 33 surgical reinterventions (bold dots) and 52 catheterization-based reinterventions (target-shaped dots) on the right ventricular outflow tract. The error bars denote $95 \%$ confidence limits as determined by the Greenwood formula. The numbers at the bottom in parentheses represent the number of patients at risk. The top row represents the surgical reintervention group; the bottom row, catheterization-based reintervention. TOF, Tetralogy of Fallot.

catheterization-based reintervention required further surgery. Of the patients undergoing surgical reintervention, $79 \%$ had undergone at least 1 catheterization-based intervention. Kaplan-Meier-estimated 6-month, 1-year, and 2-year rates of freedom from the first catheterization-based reintervention were $68 \%, 55 \%$, and $45 \%$, and those from surgical reintervention were $92 \%, 84 \%$, and $78 \%$, respectively (Figure 1 and Table 3). Of the 82 patients undergoing RV-PA conduit repair $(\mathrm{n}=44)$ or TAP $(\mathrm{n}=38)$, a significantly higher percentage of the RV-PA conduit repair group required surgical reintervention ( $46 \%$ vs $24 \%$ ).

\section{RVOT Reintervention for TOF/PS}

Among the 43 patients with TOF/PS undergoing either TAP or VSR, there was no difference in RVOT reintervention rate between the 2 groups (TAP: 11 of $24 ; 46 \%$; VSR: 7 of $19 ; 37 \%$ ) (Figure $2, A$ and $B$ ). Four patients $(21 \%)$ who underwent VSR required surgical RVOT reintervention and had a mean preoperative pulmonary valve $z$-score of -2.2 (range, -2.9 to -1.1 ), compared with 6 patients $(25 \%)$ who underwent TAP with a mean $z$-score of -3.1 (range, -3.9 to -2.5 ). These 10 patients requiring surgical RVOT reintervention had a longer median ICU LOS (10 days; IQR, 7-25 days vs 7 days; IQR, 4-10 days; $P=.028$ ) and median hospital LOS (25 days; IQR, 19-37 days vs 14 days; IQR, 9-21 days; $P<.001)$.

Kaplan-Meier-estimated 6-month, 1-year, and 2-year rates of freedom from the first catheterization-based reintervention were $50 \%, 50 \%$, and $50 \%$, respectively, for VSR and $68 \%, 68 \%$, and $57 \%$ for TAP $(P=.47$; Figure $2, A)$. Kaplan-Meier-estimated 6-month, 1-year, and 2-year rates of freedom from surgical reintervention were $85 \%, 85 \%$, and $85 \%$ for VSR and $88 \%, 88 \%$, and $82 \%$ for TAP $(P=.61$; Figure $2, B)$.

\section{RVOT Reintervention for TOF/PA}

Of the 58 patients with TOF/PA, 9 of $14(64 \%)$ who underwent TAP and 32 of $44(73 \%)$ who underwent RV-PA conduit repair required reintervention, and there were no significant differences between the 2 groups in first RVOT reintervention. Kaplan-Meier-estimated 6-month, 1-year, and 2-year rates of freedom from the first catheterizationbased reintervention were $70 \%, 51 \%$, and $40 \%$, respectively, for RV-PA conduit and $64 \%, 57 \%$, and $36 \%$ for TAP (Figure 2, C). Kaplan-Meier-estimated 6-month, 1year, and 2-year rates of freedom from surgical reintervention were $90 \%, 77 \%$, and $64 \%$ for RV-PA conduit and

TABLE 3. Catheterization and surgical event rates

\begin{tabular}{|c|c|c|c|c|}
\hline Variable & $\begin{array}{l}\text { Overall }(\mathbf{n}=101), \\
\text { n }(\%),(95 \% \text { CI })^{*}\end{array}$ & $\begin{array}{c}\text { VSR } \\
(\mathbf{n}=\mathbf{1 9}), \mathbf{n}(\%)\end{array}$ & $\begin{array}{c}\text { TAP } \\
(\mathbf{n}=\mathbf{3 8}), \mathbf{n}(\%)\end{array}$ & $\begin{array}{l}\text { RV-PA conduit } \\
(n=44), n(\%)\end{array}$ \\
\hline \multicolumn{5}{|l|}{ All RVOT (catheter/surgical) } \\
\hline \multicolumn{5}{|l|}{ Event rates } \\
\hline$<6$-mo event rate & $34(54),(42-66)$ & $7(100)$ & $12(60)$ & $13(41)$ \\
\hline 6- to 12 -mo event rate & 11 (19), (11-30) & 0 & $1(5)$ & $10(31)$ \\
\hline 1 - to 2 -y event rate & $9(15),(8-26)$ & 0 & $5(25)$ & $4(12)$ \\
\hline$>2$-y event rate & 7 (12), (6-22) & 0 & $2(10)$ & $5(16)$ \\
\hline \multicolumn{5}{|l|}{ Surgical RVOT } \\
\hline \multicolumn{5}{|l|}{ Event rates } \\
\hline$<6$-mo event rate & 7 (33), (11-37) & $2(50)$ & $1(11)$ & $4(20)$ \\
\hline 6- to 12 -mo event rate & 7 (33), (11-37) & 0 & $2(22)$ & $5(25)$ \\
\hline 1 - to 2 -y event rate & $5(15),(7-30)$ & 0 & $1(11)$ & $4(20)$ \\
\hline$>2$-y event rate & $14(42),(27-59)$ & $2(50)$ & $5(55)$ & $7(35)$ \\
\hline Death & 3/101 (2.9) & 0 & $1 / 38(2.6)$ & $2 / 44(4.5)$ \\
\hline
\end{tabular}

$C I$, Confidence interval; VSR, valve-sparing repair; $T A P$, transannular patch; $R V-P A$, right ventricle to pulmonary artery; $R V O T$, right ventricular outflow tract. *Kaplan-Meier event rate estimates. 


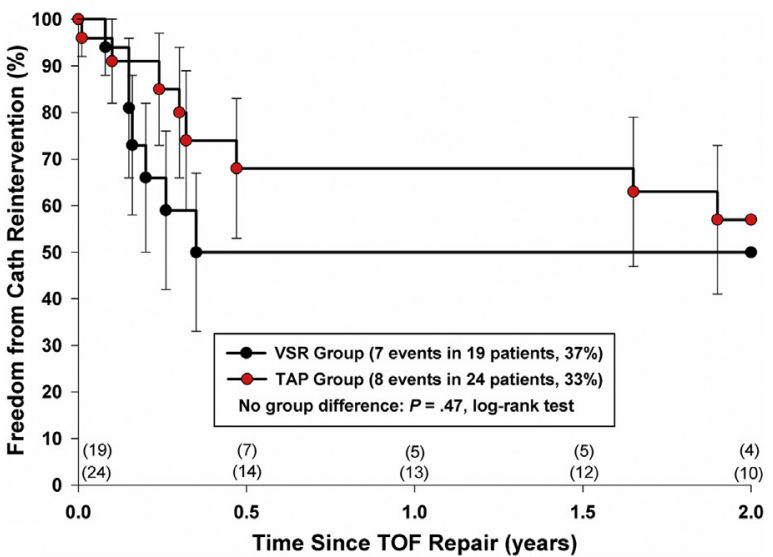

A
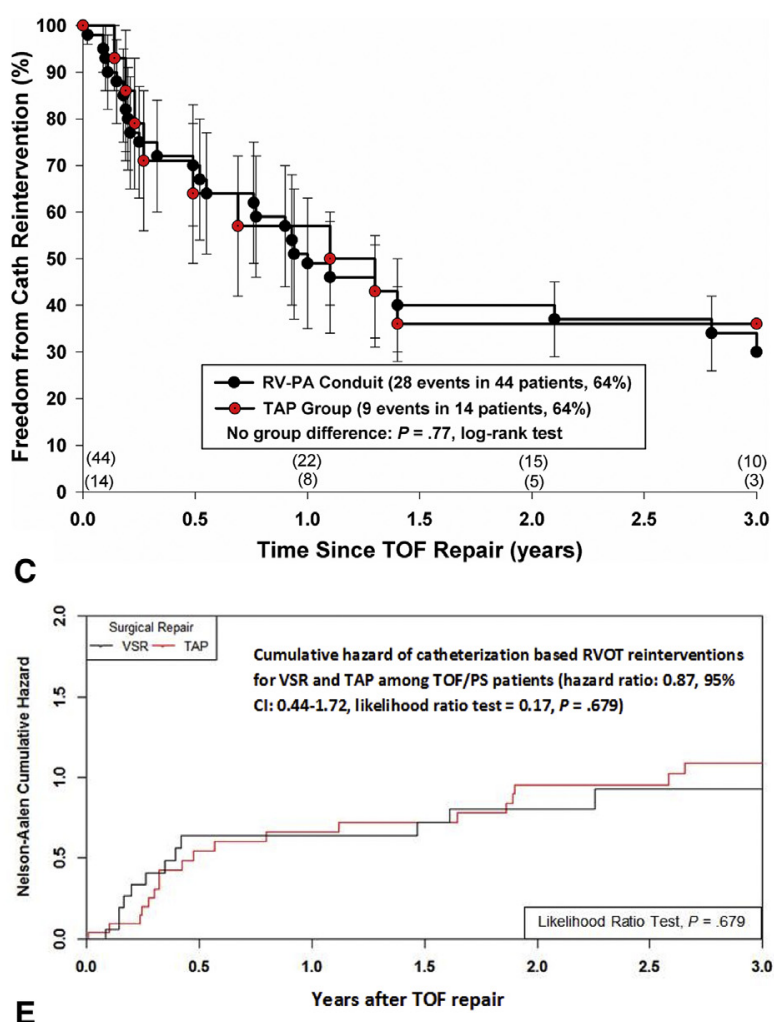

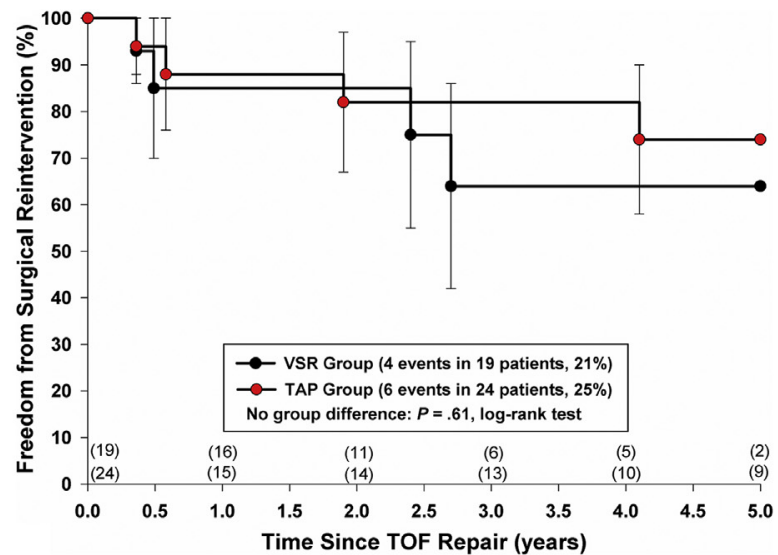

B

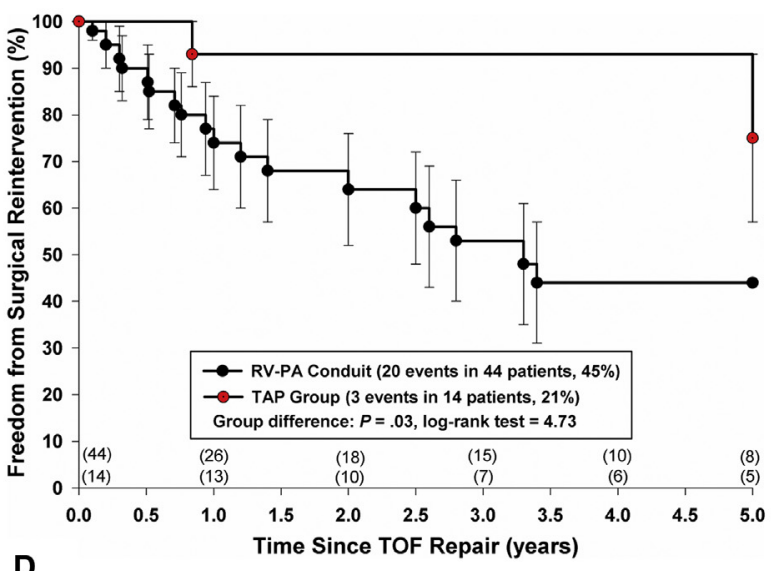

D

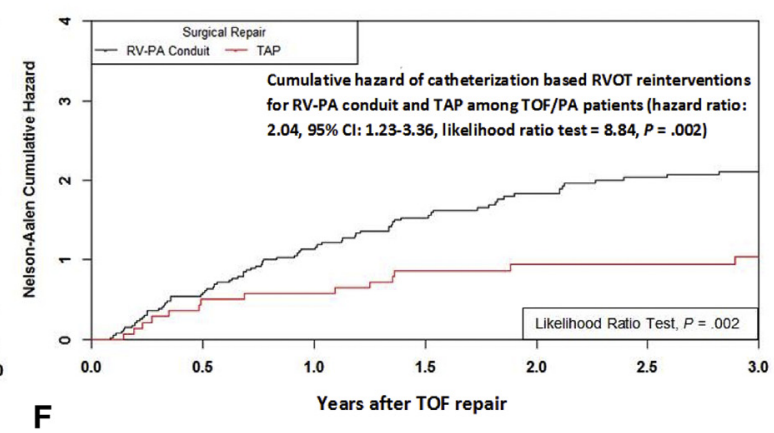

FIGURE 2. A, Kaplan-Meier curves showing freedom from first time catheterization based reintervention in patients with tetralogy of Fallot/pulmonary stenosis (TOF/PS) in the valve-sparing repair (VSR; black dots and dashes) and transannular patch (TAP; red dots and dashes) groups. The error bars denote $95 \%$ confidence limits as determined by the Greenwood formula. The numbers at the bottom in parentheses represent the number of patients at risk. The top row represents the VSR group; the bottom row, the TAP group. B, Kaplan-Meier curves showing freedom from surgical reintervention in TOF/PS patients in the VSR (black dots and dashes) and TAP (red dots and dashes) groups. The error bars denote $95 \%$ confidence limits as determined by the Greenwood formula. The numbers at the bottom in parentheses represent the number of patients at risk. The top row represents the VSR group; the bottom row, the TAP group. C, Kaplan-Meier curves showing freedom from first-time catheterization-based reintervention in patients with tetralogy of Fallot/pulmonary atresia (TOF/PA) in the right ventricle-to-pulmonary artery ( $R V-P A)$ conduit (black dots and dashes) and TAP (red dots and dashes) groups. The error bars denote $95 \%$ confidence limits as determined by the Greenwood formula. The numbers at the bottom in parentheses represent the number of patients at risk. The top row represents the RV-PA conduit group; the bottom row, the TAP group. D, Kaplan-Meier curves showing freedom from surgical reintervention in patients with TOF/PA in the RV-PA conduit (black dots and dashes) and TAP (red dots and dashes) groups. The error bars denote $95 \%$ confidence limits as determined by the Greenwood formula. The numbers at the bottom in parentheses represent the number of patients at risk. The top row represents the RV-PA conduit group; the bottom row, the TAP group. E, Nelson-Aalen cumulative hazard in patients with TOF/PS comparing the cumulative repeated catheterization based reintervention in the VSR (black) and TAP ( red) groups. F, Nelson-Aalen cumulative hazard in patients with TOF/PA comparing cumulative repeated catheterization-based reintervention in the RV-PA conduit (black) and TAP (red) groups. TOF, Tetralogy of Fallot; RVOT, right ventricular outflow tract; TOF/PA, tetralogy of Fallot/pulmonary atresia. 
TABLE 4. Catheterization-based reintervention events

\begin{tabular}{lcccc}
\hline Reintervention & Patients & Patients with $\geq \mathbf{1}$ event & Events & Events/patient \\
\hline TOF/PS & 19 & & 13 & \\
VSR & 38 & 8 & 25 & \\
TAP & & & & \\
TOF/PA & 44 & 28 & 96 & 1.042 \\
RV-PA conduit & 14 & 9 & 18 & 2.182 \\
TAP & & 8 & 1.286 \\
\hline
\end{tabular}

TOF/PS, Tetralogy of Fallot/pulmonary stenosis; VSR, valve-sparing repair; TAP, transannular patch; TOF/PA, tetralogy of Fallot/pulmonary atresia; $R V$-PA, right ventricle to pulmonary artery.

$100 \%, 93 \%$, and $93 \%$ for TAP (Figure 2, D). The median time to surgical reintervention was significantly shorter in the RV-PA conduit group (1.1 years, $95 \% \mathrm{CI}, 1$ month to 7 years) compared with the TAP group (6 years, $95 \% \mathrm{CI}$, 10 months to 6.5 years). Among the 44 patients with TOF/PA who underwent RV-PA conduit repair, 17 (39\%) had balloon dilation/stent placement and $27(61 \%)$ had surgical reintervention ( 15 with surgery on the RVOT, 5 with surgery for RVOT pseudoaneurysm, and 7 with pulmonary valve replacement).

\section{Cumulative (Repeated) Catheterization-Based RVOT Reintervention in TOF/PS and TOF/PA}

Applying modulated renewal theory with the NelsonAalen estimator analyzing all repeated reinterventions demonstrated no significant differences in the cumulative hazard for VSR and TAP among the patients with TOF/ PS (hazard ratio [HR], 0.87; 95\% CI, 0.44-1.72; likelihood ratio test $=0.17 ; P=.679$ ) (Figure $2, E$ ). In addition, our analysis revealed a significantly greater hazard for repeated events in neonates with TOF/PA undergoing RV-PA conduit replacement compared with those undergoing TAP (HR, 2.04; 95\% CI, 1.23-3.36; likelihood ratio test $=8.84$; $P=.002$ ) (Figure 2, F). Among the 28 of 44 patients with TOF/PA who had at least 1 reintervention, there were a total of 96 repeated events ( 2.182 per patient); in contrast, in the 9 of 14 patients undergoing TAP with at least 1 event, there were a total of 18 events ( 1.286 per patient) (Table 4 ). Therefore, the estimated hazard per unit time is estimated to be more than twice as high for patients with TOF/PA undergoing RV-PA conduit repair than for those undergoing TAP, indicating that a significantly greater number of RV-PA conduit catheterization- based reinterventions are needed compared with TAP for patients with TOF/PA.

\section{Surgical Reintervention Rates by Birth Weight}

When considering all patients in the study population (TOF/PA and TOF/PS), there was no difference in birth weight between those with and without surgical reintervention (median, $2.7 \mathrm{~kg}$; range, $1.3-7.6 \mathrm{~kg}$ vs $3.0 \mathrm{~kg}$; range, $1.4-$ $4.3 \mathrm{~kg}$ ). Furthermore, birth weight $<3.0 \mathrm{~kg}$ was not related to surgical reintervention in any of the TOF/PA patients
(TAP or RV-PA conduit) or TOF/PS patients (TAP only). However, patients with TOF/PS undergoing VSR who required surgical reintervention were of significantly lower median birth weight compared with those without surgical reintervention (median, $2.5 \mathrm{~kg}$; range, $1.8-3.3 \mathrm{~kg}$ vs $3.7 \mathrm{~kg}$; full range, $2.4-4.1 \mathrm{~kg}$ ). Among the $7 \mathrm{TOF} / \mathrm{PS}$ patients who underwent VSR with birth weight $<3.0 \mathrm{~kg}, 4$ $(57 \%)$ underwent surgical reintervention, compared with none of the 12 patients with birth weight $>3.0 \mathrm{~kg}(P=.005)$ (Figure 3$)$.

\section{Other Univariate Risk Factors for Reintervention}

In the whole cohort (TOF/PS and TOF/PA patients), univariate analysis indicated a significant association between a long hospital stay $(>21$ days $)(P=.003)$ and preoperative RVOT gradient $(P=.01)$. Importantly, in the subset of TOF/ PS patients, median preoperative pulmonary valve $z$-scores $(-2.6$ vs $-2.5 ; P=.53)$ and RVOT gradients (48 vs $50 \mathrm{mmHg} ; P=.45$ ) were not significantly different in patients who required surgical reintervention and those who did not.

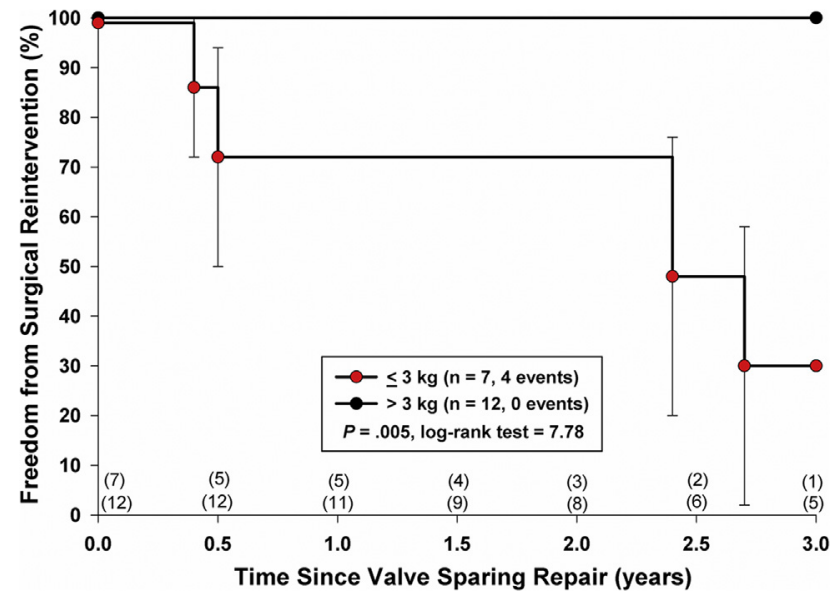

FIGURE 3. Kaplan-Meier curves showing freedom from surgical reintervention in patients with tetralogy of Fallot/pulmonary stenosis who underwent valve-sparing repair. The error bars denote $95 \%$ confidence limits as determined by the Greenwood formula. The numbers at the bottom in parentheses represent the number of patients at risk. The top row represents neonates with birth weight $<3 \mathrm{~kg}$; the bottom row, neonates $>3 \mathrm{~kg}$. 
TABLE 5. Univariate and multivariable Cox regression analysis of variables associated with reintervention

\begin{tabular}{lcc}
\hline \multicolumn{1}{c}{ Variable } & HR $(\mathbf{9 5} \% \mathbf{C I})$ & $\boldsymbol{P}$ value \\
\hline Univariate & & \\
Female vs male & $0.8(0.5-1.4)$ & .49 \\
Age $<14 \mathrm{~d}$ at surgery & $1.3(0.7-2.2)$ & .41 \\
Weight $<3 \mathrm{~kg}$ & $1.4(0.8-2.4)$ & .18 \\
Diagnosis & $0.6(0.3-1.1)$ & .08 \\
Era (before and after 2010) & $1.5(0.9-2.6)$ & .13 \\
RVOT type & & \\
$\quad$ VSR vs TAP & $0.8(0.3-2.0)$ & .67 \\
$\quad$ RV-PA conduit vs TAP & $1.3(0.6-3.0)$ & .48 \\
RVOT gradient & $0.98(0.97-0.99)$ & $.01 *$ \\
Preoperative PV $z$-score & $1.1(0.9-1.3)$ & .23 \\
ICU LOS & $1.2(0.7-2.0)$ & .63 \\
Hospital LOS & $2.3(1.3-3.0)$ & .003 \\
Multivariable & & \\
Age $<14$ d at surgery & $0.9(0.4-1.7)$ & .87 \\
Birth weight $<3$ kg & $1.7(0.9-3.2)$ & .57 \\
Era (before and after 2010) & $1.3(0.7-2.5)$ & .66 \\
Hospital LOS & $2.0(1.2-3.8)$ & .04 \\
RVOT type & & \\
VSR vs TAP & $0.81(0.33-1.94)$ & .63 \\
VSR vs RV-PA conduit & $1.3(0.57-3.0)$ & .53 \\
TAP vs RV-PA conduit & $0.62(0.35-1.09)$ & .10 \\
VSR $\times$ birth weight $<3 \mathrm{~kg} \dagger$ & $5.5(1.1-20.7)$ & $.012 *$ \\
\hline
\end{tabular}

$H R$, Hazard ratio; $C I$, confidence interval; $R V O T$, right ventricular outflow tract; $V S R$, valve-sparing repair; $T A P$, transannular patch; $R V-P A$, right ventricle to pulmonary artery; $P V$, pulmonary valve; $I C U$, intensive care unit; $L O S$, length of stay. *Statistically significant difference $(P<.05)$. $\dagger$ Significant 2 -way interaction indicating that neonates undergoing VSR with birth weight $<3 \mathrm{~kg}$ are at significant risk of reintervention.

\section{Multivariable Model for Time to Reintervention}

To adjust for possible confounders, a multivariable Cox regression model was applied to examine covariateadjusted differences in RVOT reintervention and was constructed for all patients (Table 5). In determining multivariable independent predictors of reintervention using a time-to-event Cox regression model, 5 variables-age, birth weight, era of operation, type of repair, and hospital LOSwere evaluated. Hospital LOS was an independent predictor of reintervention (HR, $2.0 ; 95 \% \mathrm{CI}, 1.2-3.8 ; P=.04$ ). The model also revealed a significant 2-way interaction between birth weight and RVOT surgical repair type $(P=.012)$, which indicated a significantly greater risk of reintervention in TOF/PS patients undergoing VSR repair at a birth weight $<3.0 \mathrm{~kg}$ (HR, 5.5; 95\% CI, 1.1-20.7).

The implication of this 2-way interaction by multivariable analysis is that a low birth weight is a significant risk factor in a time-related fashion of reintervention, but only in patients undergoing VSR and not in those undergoing other types of valve repair. Both VSR and birth weight $<3 \mathrm{~kg}$ had a significant influence on reintervention, but they are conditionally dependent. This conditional dependency is reflected in the statistically significant interaction between repair type and body weight $(P=.012)$.

\section{Cardiac ICU and Hospital LOS}

Among the 101 patients in the study population, nonparametric analysis of variance showed a comparable median ICU length of stay among the VSR, TAP, and RV-PA conduit surgical groups ( 8 days, 10 days, and 10 days, respectively; $P=.08$ ). Hospital LOS was also similar among the 3 surgical groups: VSR, 15 days; TAP, 17 days; RV-PA conduit, 18 days; $P=.03$.

\section{Survival}

Overall survival was $97 \%$, with 1 death in the TOF/PS group (a patient who underwent TAP) and 2 deaths in the TOF/PA group (patients who underwent RV-PA conduit repair). One death occurred at 11 days of age in a $1.95-\mathrm{kg}$ symptomatic TOF/PS patient with moderate bilateral lung hypoplasia, hypoplastic pulmonary arteries, large left congenital diaphragmatic hernia, and omphalocele. Following repair with TAP, the patient had suprasystemic right ventricular pressures requiring ventricular septal defect patch fenestration and extracorporeal membrane oxygenation (ECMO) support.

One of the patients with TOF/PA who died had undergone RV-PA conduit repair at 6 days of age and needed ECMO on the first postoperative day for unstable hemodynamics. Low cardiac output persisted, however, requiring significant inotropic and vasopressor support. Nonetheless, the patient deteriorated, and support was withdrawn on postoperative day 31. The second patient with TOF/PA who died also had Alagille syndrome, major aortopulmonary collateral arteries, and parachute mitral valve and underwent RV-PA conduit at 3 weeks of age. Subsequently, the patient required cardiac catheterization and balloon dilation and stenting of the proximal left pulmonary artery and the RVPA conduit. The patient underwent subsequent unifocalization of right-sided major aortopulmonary collateral arteries at age 8 months, but sustained a ventricular fibrillation arrest necessitating ECMO. He subsequently developed severe left ventricular dysfunction and resistant pseudomonas mediastinitis with septic shock and died at age 9 months.

\section{DISCUSSION}

Primary neonatal TOF repair provides several advantages, including avoidance of shunt-related complications, early relief of hypoxia, promotion of normal lung development, prevention of progressive ventricular hypertrophy and fibrosis, and psychological comfort for the family. ${ }^{17,18}$ In this series, we report excellent early outcomes and survival with complete neonatal TOF repair in patients with PS and pulmonary atresia.

There has been a shift in surgical management of TOF over the last 2 decades, with TOF repair being increasingly performed in younger patients. Nonetheless, 
the management of the hypoxic neonate with TOF remains challenging, with continuing controversy regarding the optimal initial surgical strategy to limit morbidity and mortality. There are published data supporting different approaches; however, to date no single large series has focused on primary neonatal repair.

In patients with TOF/PA, there are little reported data comparing the 2 approaches of TAP and RV-PA conduit repair. There are theoretical early and late advantages to each approach. In our series, there was no between-group difference in hospital LOS (17 days vs 18 days) or ICU LOS (10 days vs 10 days). There were more surgical reinterventions ( $45 \%$ vs $21 \%$ ) and a shorter time to reintervention (1.1 years vs 6 years) in the patients undergoing RV-PA conduit repair; however, the differences did not reach statistical significance owing to the limited number of patients and short follow-up. However, when we compared all patients with TOF/PS and TOF/PA undergoing TAP and those undergoing RV-PA conduit repair, the rate of surgical reintervention was significantly higher in RV-PA conduit patients. Patients with TOF/PA who underwent RV-PA conduit repair had a significantly greater risk of undergoing repeat catheterization-based RVOT reinterventions compared with those who underwent TAP. These reinterventions were related to conduit stenosis; however, it is important to recognize that some patients might have improved outcomes overall with an RV-PA conduit despite the increased number of RVOT reinterventions. These might include patients with anatomic or physiological defects predisposing them to higher pulmonary artery and right ventricle pressures, such as syndromic patients and those with underlying chronic lung disease. Our patients with syndromic TOF/PS with hypoplastic pulmonary arteries who underwent TAP might have had improved morbidity and survival with RV-PA conduit repair instead of TAP.

Published information on and criteria for optimal surgical repair in patients with TOF/PS are limited. In 1999, Pigula and colleagues ${ }^{19}$ reported a $3 \%$ mortality following complete repair in 99 TOF/PS patients age $<3$ months, and in 2005 , Kolcz and colleagues ${ }^{20}$ reported an early mortality of $4.5 \%$ in $45 \mathrm{TOF} / \mathrm{PS}$ patients who underwent TAP. In 2010, cumulative data from the Society of Thoracic Surgeons database showed discharge mortality of $7.8 \%$ in neonates undergoing primary TOF repair, compared with $6.2 \%$ in those undergoing the modified BT shunt procedure. $^{21,22}$ In our present series of 43 patients with TOF/PS undergoing primary neonatal TOF repair, there were no early deaths and only 1 late death, showing superior survival compared with other reported series on patients undergoing palliation with systemic to pulmonary or BT shunts. $^{21}$

Pulmonary regurgitation after TOF repair results in various long-term outcomes. ${ }^{23}$ Sousa Uva and colleagues ${ }^{24}$ reported that initial palliation with a modified BT shunt promoted pulmonary annular growth, ultimately allowing preservation of the pulmonary annulus with a valve-sparing approach (TAP in $13 \%$ with a modified BT shunt vs $56 \%$ with early complete repair; $P=.03$ ). Kanter and colleagues ${ }^{11}$ found that TOF/PS patients undergoing initial modified BT shunt were more likely to avoid a TAP compared with those who underwent primary neonatal repair $(25 \%$ vs $0 \%)$.

Although it is recognized that every effort should be made to spare pulmonary valve function as well as the pulmonary valve annulus, in patients undergoing VSR there are concerns about the need for early reintervention for residual RVOT gradients. We previously reported that residual and recurrent RVOT obstruction in patients undergoing VSR leads to RVOT reinterventions, primarily transcatheter balloon dilatation with or without stenting ${ }^{25}$; however, in our present series, $44 \%$ of the patients with TOF/PS underwent initial VSR, thereby avoiding TAP without an increase in surgical $(21 \%$ vs $25 \%)$ or catheterization-based ( $37 \%$ vs $33 \%$ ) reintervention. This difference is likely due to improvements in pulmonary valve and annular-sparing operative techniques, including the use of pulmonary valve commissurotomy with intraoperative balloon dilation of the hypoplastic pulmonary valve annulus. Robinson and colleagues $^{26}$ reported a series of $52 \mathrm{TOF} / \mathrm{PS}$ patients undergoing VSR using intraoperative pulmonary balloon valvuloplasty that showed significant pulmonary annular growth, with an increase in mean annular $z$-score from 0.57 to $1.0 .^{26}$

Although we found acceptable outcomes in neonatal TOF repair, low birth weight should ultimately factor into the decision making regarding type of RVOT repair. Curzon and colleagues ${ }^{27}$ analyzed 3022 patients using the Society for Thoracic Surgeons' congenital heart surgery database and stratification with the Risk Adjustment for Congenital Heart Surgery 1 score, they found increased surgical mortality in low-birth-weight neonates $(<2.5 \mathrm{~kg})$ with singleventricle physiology and some biventricular groups, but not in patients with TOF. In our total cohort, we found no differences in survival or reintervention outcomes, or in birth weight, between the patients undergoing VSR and those undergoing TAP. The patients who underwent TAP were slightly smaller, but there were no differences in ICU or hospital LOS, as well as no differences in the rate of surgical or catheterization-based RVOT reinterventions. However, when considering the neonates with TOF/PS undergoing VSR, lower preoperative weight ( $2.5 \mathrm{~kg}$ vs $3.7 \mathrm{~kg}$; $P=.001)$ was correlated with more frequent surgical reintervention.

\section{Study Limitations}

Our study has some limitations, including its retrospective design and single-center series. Surgeon preference 
determined the type of repair each that patient underwent and, which could have introduced potential selection bias. Some neonates with TOF/PA were more likely to undergo RV-PA conduit surgery based on a subjective analysis of underlying lung disease, difficult pulmonary artery anatomy, and underlying syndromes. Higher-risk neonates with TOF/PA were more likely to undergo RV-PA conduit repair, and thus our data might not adequately address TAP repair in these patients.

\section{CONCLUSIONS}

In summary, neonatal TOF repair can be performed with low mortality but with frequent RVOT reintervention. In neonates with TOF/PA, surgical reintervention is earlier and the rate is higher with the RV-PA conduit procedure compared with the TAP procedure. There is a significantly greater risk for repeated catheterization-based RVOT reintervention events in neonates with TOF/PA undergoing RV-PA conduit replacement compared with those undergoing TAP. Low birth weight is not associated with RVOT reinterventions in patients undergoing RV-PA conduits or TAP procedures, but is a highly significant risk factor in neonates with TOF/PS undergoing VSR. A valve-sparing approach can be performed in selected neonates with TOF/PS, but caution must be exercised when considering this approach in low-birth-weight neonates.

\section{Conflict of Interest Statement}

Authors have nothing to disclose with regard to commercial support.

\section{References}

1. Blalock A, Taussig HB. The surgical treatment of malformations of the heart in which there is pulmonary stenosis or pulmonary atresia. JAMA. 1945;128: 189-202.

2. Lillehei CW, Cohen M, Warden HE, Read RC, Aust JB, Dewall RA, et al. Direct vision intracardiac surgical correction of the tetralogy of Fallot, pentalogy of Fallot, and pulmonary atresia defects: report of first ten cases. Ann Surg. 1955;142: 418-42.

3. Castaneda AR, Freed MD, Williams RG, Norwood WI. Repair of tetralogy of Fallot in infancy: early and late results. J Thorac Cardiovasc Surg. 1977;74:372-81.

4. Barratt-Boyes BG, Neutze JM. Primary repair of tetralogy of Fallot in infancy using profound hypothermia with circulatory arrest and limited cardiopulmonary bypass: a comparison with conventional two stage management. Ann Surg. 1973; 178:406-11.

5. Lindberg HL, Saatvedt K, Seem E, Hoel T, Birkeland S. Single-center 50 years experience with surgical management of tetralogy of Fallot. Eur J Cardiothorac Surg. 2011;40:538-42.

6. Hickey EJ, Veldtman G, Bradley TJ, Gengsakul A, Manlhiot C, Williams WG, et al. Late risk of outcomes for adults with repaired tetralogy of Fallot from an inception cohort spanning four decades. Eur J Cardiothorac Surg. 2009;35: 156-64; discussion 164.
7. Reddy VM, Liddicoat JR, McElhinney DB, Brook MM, Stanger P, Hanley FL. Routine primary repair of tetralogy of Fallot in neonates and infants less than three months of age. Ann Thorac Surg. 1995;60(6 Suppl):S592-6.

8. Hirsch JC, Mosca RS, Bove EL. Complete repair of tetralogy of Fallot in the neonate: results in the modern era. Ann Surg. 2000;232:508-14.

9. Tamesberger MI, Lechner E, Mair R, Hofer A, Sames-Dolzer E, Tulzer G. Early primary repair of tetralogy of Fallot in neonates and infants less than four months of age. Ann Thorac Surg. 2008;86:1928-35.

10. Kwak JG, Lee CH, Lee C, Park CS. Surgical management of pulmonary atresia with ventricular septal defect: early total correction versus shunt. Ann Thorac Surg. 2011;91:1928-34; discussion 1934-35.

11. Kanter KR, Kogon BE, Kirshbom PM, Carlock PR. Symptomatic neonatal tetralogy of Fallot: repair or shunt? Ann Thorac Surg. 2010;89:858-63.

12. Lee CH, Kwak JG, Lee C. Primary repair of symptomatic neonates with tetralogy of Fallot with or without pulmonary atresia. Korean J Pediatr. 2014; 57:19-25.

13. Hosmer DW Jr, Lemeshow S. Applied Survival Analysis: Regression Modeling of Time to Event Data. New York: Wiley-Interscience; 1999:55-84.

14. Cox DR, Oakes D. Analysis of Survival Data. London: Chapman \& Hall; 1984: 48-57.

15. Vittinghoff E, McCulloch CE. Relaxing the rule of ten events per variable in logistic and Cox regression. Am J Epidemiol. 2007;165:710-8.

16. Kalbfleisch JD, Prentice RL. The Statistical Analysis of Failure Time Data. New York: Wiley; 1980. 32-48.

17. Pozzi M, Trivedi DB, Kitchiner D, Arnold RA. Tetralogy of Fallot: what operation, at which age. Eur J Cardiothorac Surg. 2000;17:631-6.

18. Jonas RA. Early primary repair of tetralogy of Fallot. Semin Thorac Cardiovasc Surg Pediatr Card Surg Annu. 2009;39-47.

19. Pigula FA, Khalil PN, Mayer JE, del Nido PJ, Jonas RA. Repair of tetralogy of Fallot in neonates and young infants. Circulation. 1999;100(19 Suppl):II157-61.

20. Kolcz J, Pizarro C. Neonatal repair of tetralogy of Fallot results in improved pulmonary artery development without increased need for reintervention. Eur J Cardiothorac Surg. 2005;28:394-9.

21. Al Habib HF, Jacobs JP, Mavroudis C, Tchervenkov CI, O'Brien SM, Mohammadi S, et al. Contemporary patterns of management of tetralogy of Fallot: data from the Society of Thoracic Surgeons Database. Ann Thorac Surg. 2010;90:813-9; discussion 819-20.

22. Ross ET, Costello JM, Backer CL, Brown LM, Robinson JD. Right ventricular outflow tract growth in infants with palliated tetralogy of Fallot. Ann Thorac Surg. 2015;99:1367-72.

23. Redington AN. Physiopathology of right ventricular failure. Semin Thorac Cardiovasc Surg Pediatr Card Surg Annu. 2006;3-10.

24. Sousa Uva M, Lacour-Gayet F, Komiya T, Serraf A, Bruniaux J, Touchot A, et al. Surgery for tetralogy of Fallot at less than six months of age. J Thorac Cardiovasc Surg. 1994;107:1291-300.

25. Gellis L, Banka P, Marshall A, Emani S, Porras D. Transcatheter balloon dilation for recurrent right ventricular outflow tract obstruction following valve-sparing repair of tetralogy of Fallot. Catheter Cardiovasc Interv. 2015;86:692-700.

26. Robinson JD, Rathod RH, Brown DW, Del Nido PJ, Lock JE, McElhinney DB, et al. The evolving role of intraoperative balloon pulmonary valvuloplasty in valve-sparing repair of tetralogy of Fallot. J Thorac Cardiovasc Surg. 2011; 142:1367-73.

27. Curzon CL, Milford-Beland S, Li JS, O'Brien SM, Jacobs JP, Jacobs ML, et al. Cardiac surgery in infants with low birth weight is associated with increased mortality: analysis of the Society of Thoracic Surgeons Congenital Heart Database. J Thorac Cardiovasc Surg. 2008;135:546-51.

Key Words: birth weight, pulmonary atresia, pulmonary stenosis, reintervention, right ventricle-to-pulmonary artery conduit, tetralogy of Fallot, transannular patch, valvesparing repair 